\title{
Influence of Humidity on SAW Sensor Response
}

\author{
K. JASEK* AND M. PASTERNAK \\ Military University of Technology, S. Kaliskiego 2, 00-490 Warsaw, Poland
}

\begin{abstract}
In the surface acoustic wave sensors response analysis the influence of different factors on acoustic wave propagation velocity and resonant frequency of the surface acoustic wave device plays an important role. Because the resonant frequency is commonly assumed to be a sensor response, knowing the magnitude of this influence and its mechanisms is very important. The factors which interfere the most with the surface acoustic wave sensor response include: temperature, pressure, and humidity. The influence of humidity is almost impossible to eliminate because of phenomena connected with water adsorption at the surface of the substrate as well as the electrodes of the device.
\end{abstract}

DOI: 10.12693 /APhysPolA.124.448

PACS: 43.35.Pt, 46.35.+z, 68.35.Ja

\section{Introduction}

The acoustic wave propagation velocity and the resultant resonant frequency of surface acoustic wave (SAW) is influenced by many different phenomena. Because the resonant frequency is usually measured as a sensor response, knowing the magnitude of this influence and its mechanisms is very important [1-3].

The factors which interfere the most with the response from a SAW sensor include: temperature, pressure, and humidity. The influence of humidity is the most difficult factor to eliminate because of phenomena connected with water adsorption. Unfortunately, this is true even in the case of differential SAW system sensor response measurements. This influence is significant even for strongly hydrophobic surfaces like Teflon ${ }^{\mathrm{TM}}$.

There are two mechanisms by which adsorbed water influences SAW velocity, i.e. mechanical and electrical. While the first one is connected with the mass of the adsorbed water, the second mechanism relates to an electric effect. These effects are independent of each other and may be considered separately. The theoretical research results described in [4] show that the influence of both mechanisms is similar for strong piezoelectric substrate and linearly dependent on the thickness of the layer of adsorbed water.

The SAW velocity change is given by [4]:

$$
-\frac{\Delta v}{v}=(A+B) \frac{h}{\lambda}
$$

where $A$ denotes the mechanic and $B$ denotes the electric contribution to the relative velocity change, $h$ represents the adsorbed water layer thickness that is described by an adsorption isotherm (Fig. 1). The monolayer thickness calculated from Van der Waals diameter of water molecule is $2.82 \AA$.

The components $A$ and $B$ may be expressed using simple formulae [4]:

\footnotetext{
*corresponding author; e-mail: kjasek@wat.edu.pl
}

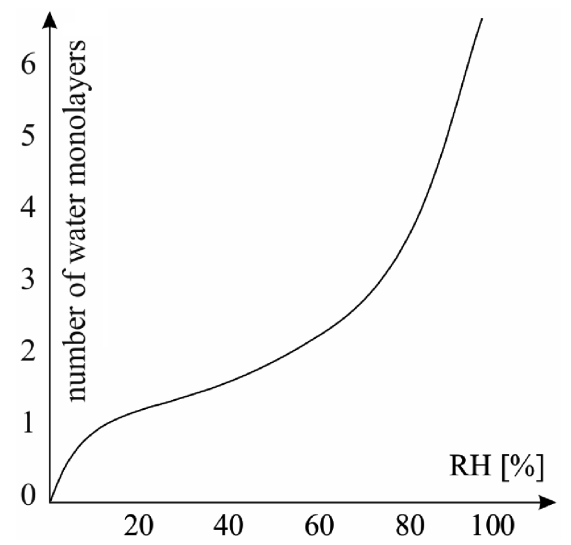

Fig. 1. Water vapour adsorption isotherm for quartz at $23^{\circ} \mathrm{C}$ [5].

$$
\begin{aligned}
& A=\frac{\rho_{\mathrm{w}}}{\rho_{\mathrm{s}}} \sqrt{1-\frac{v_{\mathrm{w}}^{2}}{v_{\mathrm{s}}^{2}}}, \\
& B=\frac{\pi k^{2} \varepsilon_{\mathrm{s}}}{\left(\varepsilon_{\mathrm{s}}+\varepsilon_{0}\right)^{2}} \frac{\varepsilon_{\mathrm{w}}^{2}+\varepsilon_{0}^{2}}{\varepsilon_{\mathrm{w}}},
\end{aligned}
$$

where $\rho_{\mathrm{w}}, \rho_{\mathrm{s}}$ are densities of water and the piezoelectric substrate, respectively, $\varepsilon_{\mathrm{w}}$ is water permittivity, $\varepsilon_{\mathrm{s}}, \varepsilon_{0}$ are substrate and vacuum permittivity, $v_{\mathrm{w}}$ is the sound velocity in water, $v_{\mathrm{s}}$ is SAW velocity and $k^{2}$ is the electromechanical coupling coefficient for the substrate.

The calculations for strong piezoelectric substrates at $200 \mathrm{MHz}$ (water permittivity depends on frequency) show that $A$ and $B$ are very similar and equal to about 0.2. For weak piezoelectric materials the situation is different. The calculations for quartz ST for the same frequency give $A \approx 0.333$ and $B \approx 0.042$. This means that the influence of an electric part of humidity interactions on weak piezoelectric substrates could practically be neglected. SAW velocity change determines the centre frequency change of SAW devices. It is easy to calculate that for a surface water film with $1 \mathrm{~nm}$ thickness the frequency change is equal to about $4.7 \mathrm{kHz}$. 
It is worth noting that the humidity contribution to the sensor response is nonlinear (like an adsorption curve) and depends on the relative humidity of the environment. The total contribution of humidity to the change of SAW velocity is bigger for the strong piezoelectric substrate, which is mainly due to the electric part of the interactions. In both cases, the influence is strong and it is necessary to take it into consideration.

\section{Experimental results}

Two kinds of quartz ST SAW resonators, i.e. one with an electrically open and another with a shorted surface, were used to make the measurements. In the first step the static impedance variation caused by relative humidity $(\mathrm{RH})$ change was measured. In Fig. 2 the results of measurements of impedance for the SAW interdigital transducer (IDT) are presented.

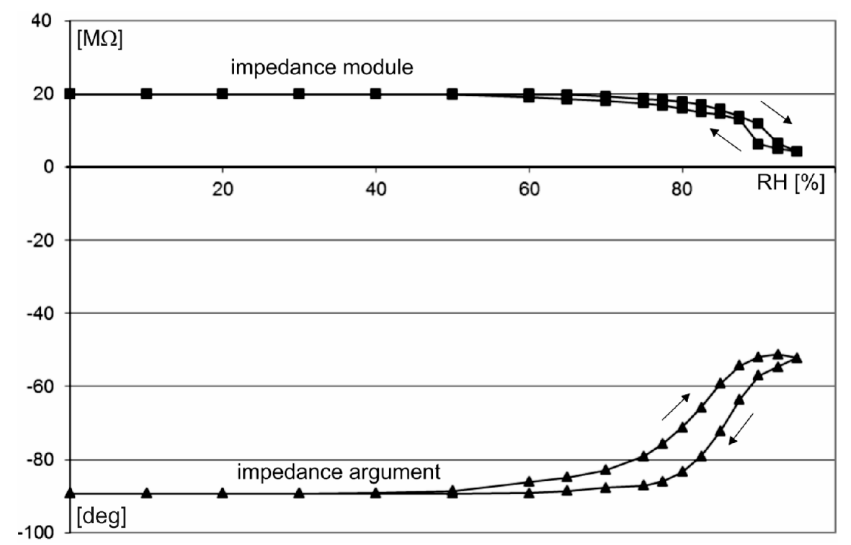

Fig. 2. Static impedance change of SAW IDT transducer (quartz ST, free surface, resonator RS197 [6]).

It can be noted that both the module and the argument of the static impedance have some hysteresis. Noticeable changes of those parameters start at $50 \%$ of $\mathrm{RH}$ and significant changes are visible at about $80 \%$ of $\mathrm{RH}$. It is consistent with the character of the adsorption isotherm in Fig. 1. For RH values close to saturation the centre frequency changes are much higher than those calculated using formula (1). This is caused by the strong additional effects connected with the IDT electric parameter changes. The SAW resonator frequency changes caused by humidity have a very similar character. The measurements were carried out using an experimental setup described in [7] where two kinds of SAW resonators RS197 were measured. The surface of the first one was shorted by a film of gold which was deposited on it while the surface of the second resonator remained free. The golden layer practically eliminated the electrical influence of the water layer on SAW velocity.

In Figs. 3 and 4 some exemplary results are shown. In the measurement presented in Fig. 3 the $\mathrm{RH}$ was changed from 0 to $80 \%$ and then back to 0 again. Figure 4 shows the concentration characteristic for this measurement. It is interesting to note that the resonant frequency changes are very similar for both substrates. A significant difference appears for very high humidity ( $>80 \% \mathrm{RH}$ ), which can be attributed to the different condensation of water vapour on quartz and gold surfaces. The change amounts to $8 \mathrm{kHz}$ and is consistent with the calculated results $(\approx 4.7 \mathrm{kHz}$ per $\mathrm{nm}$ of water $)$.

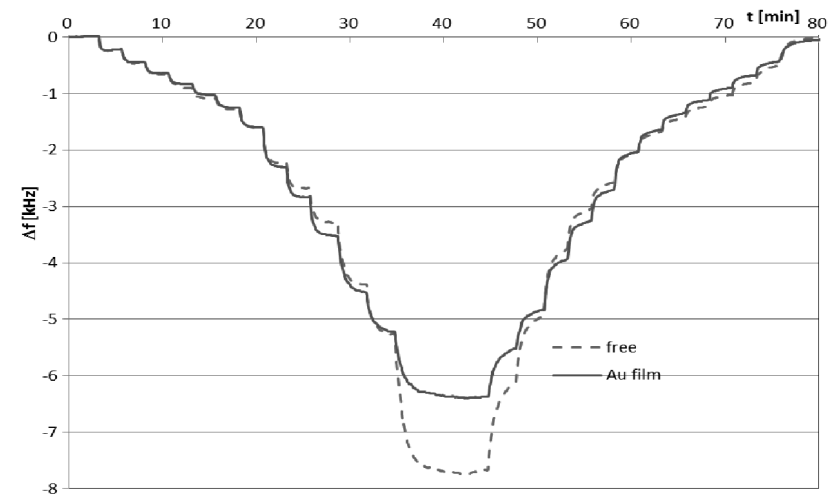

Fig. 3. Influence of humidity on centre frequency changes of shorted (Au film) and open SAW resonators. Visible steps correspond with an RH change from 0 to over $80 \%$.

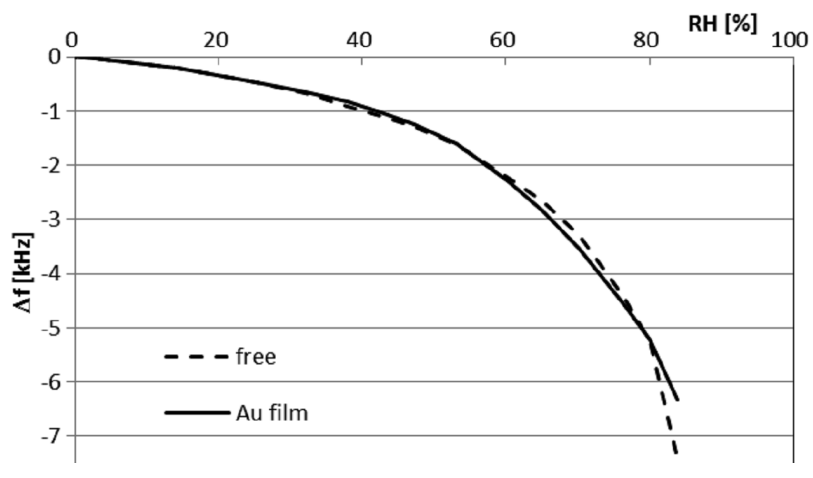

Fig. 4. Influence of humidity on centre frequencies of measured resonators.

It can be observed that the mechanical part of Eq. (1) dominates regardless of surface state and that is determined by the shape of an adsorption isotherm. Actually the differences between two curves from Fig. 4 are not very big and their character is very similar. In general, it can be concluded that each SAW sensor (regardless of the kind of deposited chemosensitive film) is also an $\mathrm{RH}$ sensor. However, the conclusion is only valid for ambient temperatures because the adsorbed water layer thickness decreases strongly when the temperature increases. This is clearly visible in the measurement results shown in Fig. 5.

The water vapour influence for a SAW device centre frequency can therefore be reduced many times if this device works in a higher temperature. 


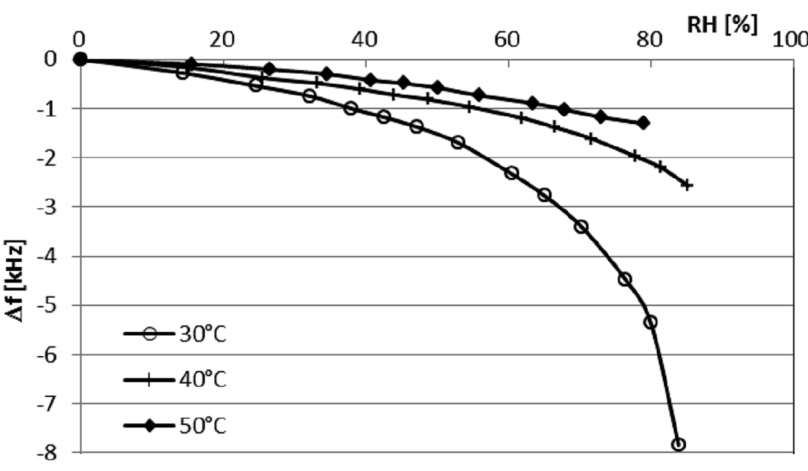

Fig. 5. Frequency changes for free quartz SAW device at different temperatures.

\section{Conclusions}

Based on the calculation and measurements that were carried out it can be concluded that the main mechanisms by which water vapour influences the quartz SAW devices centre frequency is mass loading. For $\mathrm{RH}$ values over $50 \%$ the electrical effect starts to play a noticeable role because of water influence on IDT parameters. For RH values close to saturation this mechanism is significant.

The frequency change and thereby the SAW sensor response to the $\mathrm{RH}$ change is determined by the adsorption isotherm for a given substrate, which is strongly dependent on the temperature. The change is about $4.7 \mathrm{kHz}$ for each nm of water layer thickness or about four layers of water molecules [8]. The results of the measurement of the effects of frequency change for free and shorted quartz substrates caused by a change of $\mathrm{RH}$ are very similar. This allows us to conclude that the degree of water adsorption for free and gold coated quartz sub- strates within a low and middle humidity range is almost the same.

The results presented here confirm the simple theoretical model described in [4]. They show clearly that each SAW device, even a filmless one, is very sensitive to water vapour. This sensitivity increases with the electromechanical coupling coefficient of piezoelectric substrates and it is undesirable in most applications of SAW sensors. In this respect the quartz substrate seems to perform better than other strong piezoelectric substrates (e.g. lithium niobate).

The dependence of SAW sensor response on water layer thickness makes the possibility of humidity influence compensation in differential systems debatable in many cases, given that the adsorption isotherms are specific for different sensing coatings.

\section{References}

[1] W. Jakubik, M. Urbanczyk, E. Maciak, T. Pustelny, Bull. Pol. Acad. Sci., Techn. Sci. 56, 133 (2008).

[2] E. Maciak, Z. Opilski, T. Pustelny, M. Bednorz, J. Phys. IV (France) 129, 131 (2005).

[3] T. Pustelny, J. Ignac-Nowicka, B. Jarzabek, A. Burian, Opt. Appl. 34, 551 (2004).

[4] G.B. Dorjin, I.G. Simakov, Acoust. Phys. 48, 436 (2002).

[5] Y. Awakuni, J.H. Calderwood, J. Phys. D, Appl. Phys. 5, 1038 (1972).

[6] W. Soluch, IEEE Trans. Ultrason. Ferroelect. Freq. Contr. 55, 1391 (2008).

[7] K. Jasek, W. Miluski, M. Pasternak, Acta Phys. Pol. A 124, 445 (2013).

[8] D.B. Asay, S.H. Kim, J. Phys. Chem. B 109, 16760 (2005). 